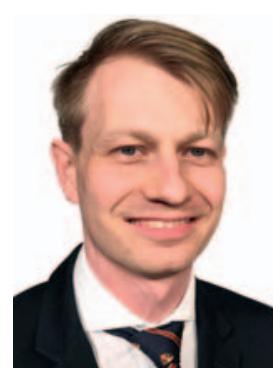

Sebastian Zimmer

Koordination «DermaCampus»

\title{
Wissenswertes rund um die Diagnostik
}

Herzlich willkommen zur vierten Ausgabe des «DermaCampus» in diesem Jahr! Auch dieses Mal finden Sie darin wieder praxisrelevante Inhalte, die für Ihre Facharztweiterbildung nützlich sind.

Der Blick auf die Psoriasis von einer Hauterkrankung zu einer Systemerkrankung hat sich in den letzten Jahren dank unaufhörlicher Forschung weiterentwickelt. Eine häufige Manifestation der systemischen Inflammation stellt die Psoriasis-Arthritis dar. Die Dermatologie hat hier eine verantwortungsvolle Stellung. Wir müssen die Patienten aktiv nach einer Gelenkbeteiligung bei Psoriasis befragen, um die Diagnose stellen zu können. Den meisten Patienten ist der Zusammenhang zwischen Haut und Gelenken bei der Schuppenflechte jedoch nicht bekannt. Dr. Charlotte Krausewitz, Assistenzärztin der Rheumatologischen Abteilung des Universitätsklinikums Bonn hat einen prägnanten Artikel zur Diagnostik der Psoriasis-Arthritis verfasst.

Neben Standardliteratur greifen Kolleginnen und Kollegen zur Vorbereitung auf die Facharztprüfung auch auffachspezifische Leitlinien zurück. Im Versorgungsalltag sind Leitlinien in Zeiten der evidenzbasierten Medizin fester Bestandteil, da man hier die aktuellen Diagnose- und Therapieempfehlungen schnell findet. Aber was steckt hinter dem «Mythos Leitlinie»? Einen Einblick in deren Entstehung - von der Notwendigkeit bis zur Implementierung - gibt uns PD Dr. Thomas Eigentler aus Tübingen anhand der kürzlich überarbeiteten S3-Leitlinie Melanom.

Zusätzlich enthält diese Ausgabe eine interessante Blickdiagnose und eine Buchrezension zum Nachfolgerbuch des Standardwerks der Dermatoskopie von Prof. Wilhelm Stolz «Bildgebende Diagnostik in der Dermatologie». Die Rezension wurde von Dr. Clara Geppert, die sich im dritten Weiterbildungsjahr zur Dermatologin befindet, verfasst. Die Blickdiagnose stammt aus der Feder von Dr. Anup Kumar Tiwary.

Wir wünschen Ihnen eine anregende Lektüre! Mit besten Grüßen,

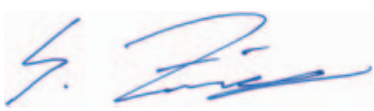

\section{KARGER}

Fax +4976145207 14 information@karger.com www.karger.com (c) 2018 S. Karger GmbH, Freiburg
Dr. Sebastian Zimmer 
Differentialdiagnosen im Überblick

Psoriasis-Arthritis

Die Psoriasis-Arthritis (PsA) ist eine autoimmunvermittelte, in der Regel Rheumafaktor-negative, entzündlich-rheumatische Systemerkrankung [1]. Die Arthritis stellt sich in der Mehrzahl der Fälle erst nach dem Auftreten psoriatischer Hautveränderungen ein. Etwa $47 \%$ der Patienten mit PsA weisen bereits zwei Jahre nach Erstmanifestation irreversible strukturelle Gelenkdestruktionen auf, sodass eine frühzeitige Diagnosestellung und Therapieeinleitung von entscheidender Bedeutung ist [2,3].

\section{Epidemiologie und Ätiologie}

5-40\% der Psoriasis-Patienten sind im Rahmen ihrer Grunderkrankung von einer PsA betroffen [4-8]. Bezüglich der Inzidenz und Prävalenz ist sich die Literatur bislang uneinig. Eine neuere Studie beziffert die jährliche Inzidenz unter den Patienten mit Psoriasis vulgaris auf 23\% [9]. Offenbar besteht eine Korrelation zwischen der Anzahl an betroffenen Hautarealen und dem Risiko, an einer PsA zu erkranken [1].

Die Ätiologie ist bisher weitgehend ungeklärt; es wird ein Zusammenspiel von genetischen, immunologischen und Umweltfaktoren vermutet [10-12].

\section{Klassifikationen}

Entsprechend den Gelenkmanifestationen werden nach Moll und Wright fünf Untergruppen unterschieden [13, 14]:

- Asymmetrische Oligoarthritis (15-50\%)

- Symmetrische Polyarthritis (30-80\%; ähnlich der rheumatoiden Arthritis)

- Distale interphalangeale Arthritis (5-15\%)

- Mutilierende Arthritis (ca. 5\%)

- Wirbelsäulenbeteiligung (5-40\%; Korrelation mit HLA-B27)

In der klinischen Anwendung zeigt sich, dass die beschriebenen Gruppen lediglich im Rahmen der Frühdiagnose gegeneinander abgegrenzt werden können, im Krankheitsverlauf jedoch häufig ineinander übergehen oder parallel auftreten [14]. Daher beschränkt man sich zumeist auf die Einteilung in axiale (Sakroiliitis mit oder ohne periphere Arthritis) und periphere Manifestation (ohne Sakroiliitis), wobei erstere nur etwa ein Drittel der Fälle ausmacht [15].

Da bislang keine Diagnosekriterien existieren, behilft man sich im klinischen Alltag zumeist mit den von Taylor et al. 2006 publizierten «Classification criteria for psoriatic arthritis»(CASPAR)Kriterien [16] (Tab. 1). Hierbei handelt es sich um Klassifikationskriterien, die insbesondere der Abgrenzung gegenüber anderen entzündlichen und degenerativen Gelenkerkrankungen dienen. Voraussetzung ist eine gesicherte entzündliche Manifestation an peripheren Gelenken, der Wirbelsäule oder Sehnen. Treffen zusätzlich mindestens drei der aufgeführten Nebenkriterien zu, so kann die Diagnose einer PsA mit einer Spezifität von nahezu 99\% gestellt werden. Schwachpunkt der CASPAR-Kriterien ist sicherlich die mangelnde Sensitivität, sodass die Diagnosestel-

\section{Tab. 1. CASPAR-Kriterien zur Klassifikation der Psoriasis-Arthritis}

Die Klassifikationskriterien sind erfüllt bei Vorliegen einer entzündlichen Erkrankung der Gelenke, der Wirbelsäule und/oder der Sehnen/Sehnenansätze und einem Score von mindestens 3 Punkten aus folgenden Kategorien:

\begin{tabular}{ll}
\hline & Punkte \\
\hline $\begin{array}{l}\text { Nachweis einer Psoriasis } \\
\text { • Bestehende Psoriasis } \\
\text { • Anamnestisch bekannte Psoriasis } \\
\text { • Positive Familienanamnese }\end{array}$ & $\begin{array}{l}\text { 2 oder } \\
1 \text { oder } \\
1\end{array}$ \\
\hline $\begin{array}{l}\text { Psoriatische Nagelbeteiligung } \\
\text { • Tüpfelung, Onycholyse, Hyperkeratose }\end{array}$ & 1 \\
\hline $\begin{array}{l}\text { Rheumafaktor negativ } \\
\text { Daktylitis }\end{array}$ & 1 \\
• Bestehende Daktylitis (Finger oder Zehe) & 1 oder \\
\hline $\begin{array}{l}\text { Radiologische Zeichen einer gelenknahen } \\
\text { Knochenneubildung }\end{array}$ & 1 \\
\hline
\end{tabular}

lung letztlich von der Expertise des behandelnden Arztes abhängig ist [17].

\section{Klinisches Bild}

Die PsA äußert sich zumeist als schmerzhafte Oligo- oder Polyarthritis mit synovitischen Schwellungen, teils auch Rötungen der betroffenen Gelenke. Charakteristisch sind der Befall der Fingerund Zehenendgelenke (Transversal-Arthritis), häufig mit begleitender Nagelbeteiligung, sowie der Befall «im Strahl» als gleichzeitiger Befall von Grund-, Mittel- und Endgelenk. Betroffene Patienten berichten vielfach über Morgensteifigkeit, schmerzbedingtes nächtliches Erwachen sowie Funktionseinschränkungen. Die häufig zu beobachtende Beteiligung des Achsenskeletts, insbesondere der Iliosakralgelenke, mit HLA-B27-Assoziation ist maßgeblich für die Eingliederung der PsA in die Gruppe der Spondyloarthritiden. Neben der Gelenkaffektion liegen regelmäßig Sehnen- und Weichteilmanifestationen in Form von Enthesitis, Insertionstendinitis und Daktylitis («Wurstfinger», «Wurstzehe») vor. Seltener werden begleitende Iritis, orale Ulzerationen und Urethritis beobachtet.

In der dermatologischen Anamnese und klinischen Untersuchung sollten explizit Nacht- und Ruheschmerz sowie Nagelbeteiligung geprüft werden. Eine Daktylitis ist nahezu beweisend für die PsA und somit eine Blickdiagnose (Abb. 1a, b).

\section{Diagnostik}

Auf Grundlage der klinischen Untersuchung gestaltet sich die Beurteilung einer entzündlichen Gelenk-, Sehnen- oder Wirbelsäulenmanifestation oft schwierig, sodass bildgebenden Verfahren wie konventionellem Röntgen sowie insbesondere Magnetresonanz- 
Abb. 1a, b. Blickdiag nose einer typischen Daktylitis bei PsA Betroffen sind hier Dig. man. II und Dig. ped. II und III, jeweils rechts im Bild.

(c) Dr. MUDr. Valentin Schäfer
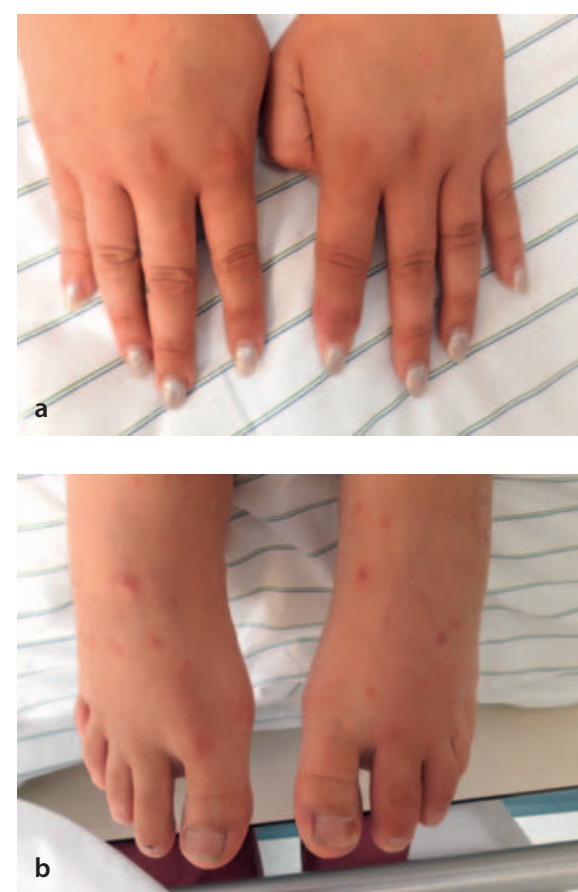

b

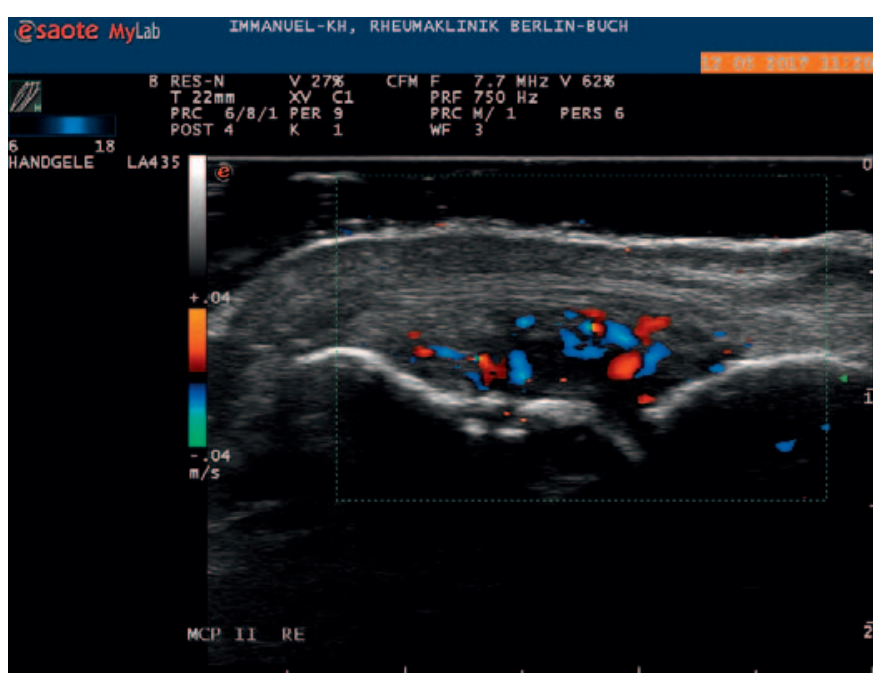

Abb. 2. Darstellung eines Metacarpophalangealgelenk(MCP)-Gelenks im Longitudinalschnitt bei einer Patientin mit PsA. Auffällig sind Hyperperfusion im Farbdoppler, Kapselabhebung und Synovialproliferation als Zeichen der entzündlichen Gelenkaktivität. @ Dr. MUDr. Valentin Schäfer

lung zur interdisziplinären Zusammenarbeit und Therapieentscheidung.

Im Falle von Arthralgien ohne klinisch objektivierbare Arthritiszeichen ist weiterhin eine symptomorientierte Therapie mit nichtsteroidalen Antirheumatika und Cyclooxygenase(COX)-Inhibitoren angeraten.

Bei anamnestischem, klinischem oder bildgebendem Nachweis einer Arthritis oder Daktylitis wird die Einleitung einer Basistherapie zur Vorbeugung bzw. Reduktion destruktiver Gelenkveränderungen empfohlen. Hier sollten zunächst konventionelle DMARDs (Disease-modifying anti-rheumatic drugs) Einsatz finden, die eine gute Gelenk- und Hautwirksamkeit versprechen (z.B. Methotrexat s.c.). Bei Refraktärität oder Kontraindikationen der Erstlinientherapie wird die Medikation mit biologischen DMARDs (Adalimumab, Certolizumab, Etanercept, Golimumab, Infliximab, Secukinumab, Ustekinumab) als Mono- oder Kombinationstherapie mit einem synthetischen DMARD (Methotrexat, Leflunomid) empfohlen. Biologische DMARDs reduzieren nachweislich das Fortschreiten der peripheren Gelenkzerstörung [24-27]. Die Auswahl eines geeigneten Präparats (Tumornekrosefaktor(TNF)-a-Blocker, Interleukin(IL)-Inhibitoren usw.) ist dem behandelnden Arzt freigestellt; Wirksamkeitsvorteile existieren kaum. Bei axialer Manifestation ist die Gruppe der Biologika den synthetischen DMARDs deutlich überlegen, sodass letztere bei entsprechender Symptomatik nicht als Monotherapeutikum eingesetzt werden sollten. Stehen Arthritis, Daktylitis und gegebenenfalls Enthesitis bei gleichzeitigem psoriatischem Hautbefall im Vordergrund, so ist Apremilast eine gute Alternative zur Therapie mit biologischen DMARDs [28].

Glukokortikoide sind nur zur überbrückenden Therapie bis zum verzögerten Wirkungseintritt der DMARDs sowie zur intraartikulären Injektion bei Mon- oder Oligoarthritis geeignet; hierbei sollten nicht mehr als zwei Gelenke gespritzt werden. Eine Dauertherapie sollte aufgrund des ausgeprägten Nebenwirkungsprofils vermieden werden. 


\section{Fazit für die Praxis}

Die PsA ist eine vielfach erosiv-proliferativ verlaufende Gelenkerkrankung, die mit einer erheblichen Funktionseinschränkung und Minderung der Lebensqualität einhergehen kann. Da die Psoriasis der Haut in der Mehrzahl der Fälle einer Gelenkmanifestation vorausgeht, liegt es in der Verantwortung der behandelnden Dermatologen und Hausärzte, Aufklärung und Screening zu betreiben und Frühzeichen zu erkennen. Bei Verdacht auf Vorliegen einer PsA sollte eine zeitnahe rheumatologische Mitbeurteilung erfolgen, um eine frühzeitige Diagnosestellung und Therapieeinleitung zu gewährleisten. Dies ist an unserer Institution durch eine monatliche interdisziplinäre Sprechstunde mit den Kollegen der Dermatologie gegeben.

\section{Literatur}

1. Krome S: Wie häufig ist die assoziierte Arthritis bei Psoriasis-Patienten? Kompendium Rheumatologie 2009;5:24.

2. Kane D, Pathare S: Early psoriatic arthritis. Rheum Dis Clin North Am 2005;31: 641-657.

3. Reddy SM, Anandarajah AP, Fisher MC, et al.: Comparative analysis of disease activity measures, use of biologic agents, body mass index, radiographic features, and bone density in psoriatic arthritis and rheumatoid arthritis patients followed in a large U.S. disease registry. J Rheumatol 2010;37:2566-2572.

4. Helliwell PS, Taylor WJ: Classification and diagnostic criteria for psoriatic arthritis. Ann Rheum Dis 2005;64(suppl 2):ii3-8.

5. Köhm M, Behrens F: Diagnosis and therapy of early psoriatic arthritis. Z Rheumato 2012;71:46-52.

6. Sadek HA, Abdel-Nasser AM, El-Amawy, Hassan SZ: Rheumatic manifestations of psoriasis. Clin Rheumatol 2007;26:488-498.

7. Wong K, Gladman DD, Husted J, et al.: Mortality studies in psoriatic arthritis: results from a single outpatient clinic. I. Causes and risk of death. Arthritis Rheum 1997;40:1868-1872.

8. Zachariae $\mathrm{H}$ : Prevalence of joint disease in patients with psoriasis: implications for therapy. Am J Clin Dermatol 2003;4:441-447.

9. Ritchlin CT, Colbert RA, Gladman DD: Psoriatic Arthritis. N Engl J Med 2017;376: 957-970.

10. Eder L, Chandran V, Pellet F, et al.: Human leucocyte antigen risk alleles for psoriatic arthritis among patients with psoriasis. Ann Rheum Dis 2012;71:50-55.

11. Haroon M, Winchester R, Gilles JT, et al.: Certain class I HLA alleles and haplotypes implicated in susceptibility play a role in determining specific features of the psoriatic arthritis phenotype. Ann Rheum Dis 2016;75:155-162.

12. Ritchlin C: Psoriatic disease - from skin to bone. Nat Clin Pract Rheumatol 2007;3: 698-706.

13. Moll JM, Wright V: Psoriatic arthritis. Semin Arthritis Rheum 1973;3:55-78.
14. Gladman DD, Antoni C, Mease P, et al.: Psoriatic arthritis: epidemiology, clinical features, course, and outcome. Ann Rheum Dis 2005;64(suppl 2):ii14-17.

15. Marsal S, Armadans-Gil L, Martinez M, et al.: Clinical, radiographic and HLA associations as markers for different patterns of psoriatic arthritis. Rheumatology (Oxford) 1999;38:332-337.

16. Taylor W, Gladman D, Helliwell P, et al.: Classification criteria for psoriatic arthritis: development of new criteria from a large international study. Arthritis Rheum 2006; 54:2665-2673.

17. McArdle A, Pennington S, FitzGerald O: Clinical Features of Psoriatic Arthritis: a Comprehensive Review of Unmet Clinical Needs. Clin Rev Allergy Immunol 2017 DOI: $10.1007 / \mathrm{s} 12016-017-8630-7$.

18. Schäfer VS, Fleck M, Kellner H, et al.: Evaluation of the novel ultrasound score for large joints in psoriatic arthritis and ankylosing spondylitis: six month experience in daily clinical practice. BMC Musculoskelet Disord 2013;14:358.

19. Alenius GM, Berglin E, Rantapää Dahlqvist S: Antibodies against cyclic citrullinated peptide (CCP) in psoriatic patients with or without joint inflammation. Ann Rheum Dis 2006;65:398-400.

20. Härle P, Hartung W, Lehmann P, et al.: Detection of psoriasis arthritis with the GEPARD patient questionnaire in a dermatologic outpatient setting. Z Rheumatol 2010;69:157-160, 162-163.

21. Coates LC, Murphy R, Helliwell PS: New GRAPPA recommendations for the management of psoriasis and psoriatic arthritis: process, challenges and implementation. Br J Dermatol 2016;174:1174-1178

22. Gossec L, Coates LC, de Wit M, et al.: Management of psoriatic arthritis in 2016: a comparison of EULAR and GRAPPA recommendations. Nat Rev Rheumatol 2016; 12:743-750.

23. Gossec L, Smolen JS, Ramiro S, et al.: European League Against Rheumatism (EULAR) recommendations for the management of psoriatic arthritis with pharmacological therapies: 2015 update. Ann Rheum Dis 2016;75:499-510.

24. Antoni C, Krueger GG, de Vlam K, et al.: Infliximab improves signs and symptoms of psoriatic arthritis: results of the IMPACT 2 trial. Ann Rheum Dis 2005;64:11501157.

25. Kavanaugh A, McInnes I, Mease P, et al.: Golimumab, a new human tumor necrosis factor alpha antibody, administered every four weeks as a subcutaneous injection in psoriatic arthritis: Twenty-four-week efficacy and safety results of a randomized, placebo-controlled study. Arthritis Rheum 2009;60:976-986.

26. Mease PJ, Gladman DD, Ritchlin CT, et al.: Adalimumab for the treatment of patients with moderately to severely active psoriatic arthritis: results of a double-blind randomized, placebo-controlled trial. Arthritis Rheum 2005;52:3279-3289.

27. Mease PJ, Kivitz AJ, Burch FX, et al.: Etanercept treatment of psoriatic arthritis: safety, efficacy, and effect on disease progression. Arthritis Rheum 2004;50:2264-2272.

28. Nast A, Amelunxen L, Augustin M, et al.: Arbeitsgemeinschaft der Wissenschaftlichen Medizinischen Fachgesellschaften: S3-Leitlinie zur Therapie der Psoriasis vulgaris (Update 2017). Registernummer 013/001. Stand 10/2017.

Kontaktadresse: Dr. Charlotte Krausewitz, Dr. MUDr. Valentin S. Schäfer, Rheumatologische Abteilung, Medizinische Klinik III, Hämatologie, Onkologie, Rheumatologie, Universitätsklinikum Bonn, Sigmund-FreudStraße 25, 53127 Bonn, Deutschland

\section{Experte im Gespräch \\ Die Entstehung von S3-Leitlinien}

Damit die S3-Leitlinie Melanom wieder auf dem aktuellen Stand ist, wurde sie gerade überarbeitet und im Juli 2018 fertiggestellt. Leitlinien sind aus dem Praxisalltag nicht wegzudenken, aber wie genau entstehen sie denn? PD Dr. Thomas Eigentler erläutert im folgenden Interview die einzelnen Schritte und was bei der Erstellung bzw. Überarbeitung unbedingt berücksichtigt werden muss.

\section{Wer entscheidet, dass eine Leitlinie erstellt werden soll?}

Eine Fachgesellschaft kann bei der Arbeitsgemeinschaft der Wissenschaftlichen Medizinischen Fachgesellschaften e.V. (AWMF), der aktuell 178 Fachgesellschaften angehören, ein neue Leitlinie anmelden. Dieser Anmeldeprozess ist strukturiert und enthält Prüf- mechanismen, sodass z.B. eine Leitlinie nicht von mehreren Fachgesellschaften parallel entwickelt wird. Bei der S3-Leitlinie zum Melanom hat die Deutsche Dermatologische Gesellschaft e.V. (DDG) und die Deutsche Krebsgesellschaft e.V. (DKG) einen solchen Antrag gestellt und das entsprechende Mandat erhalten.

\section{Was ist der erste Schritt bei der Entwicklung einer Leitlinie?}

Der erste Schritt bei der Entwicklung ist die möglichst umfassende Ansprache anderer Fachgesellschaften, Arbeitsgemeinschaften und Patientenvertretungen und die Formulierung der Schlüsselfragen. Die Schlüsselfragen sind der Grundstein für die evidenzbasierte Literaturrecherche, die die Grundlage für die Hintergrund- 
texte und somit auch für die Empfehlungen der Leitlinie sind. In einer ersten Konferenz werden die Schlüsselfragen von den Mandatsträgern (Abgesandte der Fachgesellschaften, Arbeitsgemeinschaften und Patientenvertretungen) verabschiedet und die Arbeitsgruppen für die Erarbeitung der Hintergrundtexte und Empfehlungen gebildet.

\section{Wie wird die Entwicklung von Leitlinien finanziert?}

Die Finanzierung der Leitlinien ist industrieunabhängig. Bei der S3-Leitlinie zum Melanom erfolgte die Finanzierung über das Leitlinienprogramm Onkologie der DKG, welches wiederum durch die Deutsche Krebshilfe mitfinanziert wird. Alle Beteiligten müssen übrigens Ihre Interessenkonflikte umfassend angeben. Diese sind im Leitlinienreport, der jeder Leitlinie beiliegt, nachzulesen. Dort ist auch vermerkt, wie mit Mandatsträgern umgegangen wird, wenn bei einer Empfehlung ein Interessenkonflikt besteht.

\section{Wie lange dauert die Entstehung einer Leitlinie?}

Für die erste Version wurde eine Entwicklungszeit von 3 Jahren veranschlagt. Aktualisierungen benötigen erfahrungsgemäß weniger Zeit. Für die S3-Leitlinie Melanom streben wir ein Update pro Jahr an, welches allerdings nicht alle Kapitel revidiert, sondern jene, bei denen es auch einen Aktualisierungsbedarf gibt.

\section{Ist die Gültigkeitsdauer einer Leitlinie von vorneherein festgelegt?}

Die Gültigkeitsdauer ist durch die Statuten der AWMF festgelegt und beträgt in der Regel 3 Jahre. Die Frist zur Aktualisierung kann jedoch gegebenenfalls verlängert werden, sodass ausgewählte Leitlinien auch eine längere Gültigkeit haben können.

\section{Wie viele Experten arbeiten an einer Leitlinie und wie wird die Arbeit organisiert?}

Je nach Umfang der Leitlinie arbeiten sehr viele Experten an einer Leitlinie mit. Bei der S3-Leitlinie Melanom waren es im zeitlichen Verlauf sicherlich über 70 Personen, die mitgearbeitet haben.

Die Entwicklung läuft in mehreren Schritten ab. Eine kleinere Anzahl von Personen führt die Literaturrecherche für jede Schlüssel- frage durch und bewertet mit Fachexperten die gefundene Literatur. Die Volltexte werden dann systematisch aufgearbeitet und nach Ihrer Güte klassifiziert. Diese Ergebnisse finden sich in den Evidenztabellen wieder, die Grundlage für die Hintergrundtexte sind und damit auch die Argumentation für die Empfehlungen darstellen. Die Empfehlungen werden dann in einer Konsensuskonferenz von den Mandatsträgern konsentiert. Oftmals werden die Empfehlungstexte der Arbeitsgruppen in der Konsensuskonferenz nochmals eindeutiger formuliert oder besser an die klinische Situation angepasst. Eine Empfehlung ist angenommen, wenn dieser mindestens 75\% der Mandatsträger zustimmen.

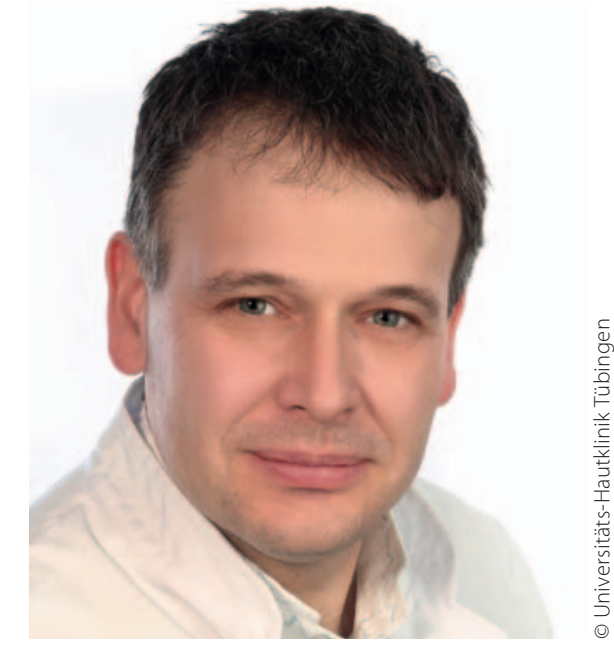

«Die Schlüsselfragen sind der Grundstein für die evidenzbasierte Literaturrecherche, die die Grundlage für die Hintergrundtexte und somit auch für die Empfehlungen der Leitlinie sind.»
Wie wird eine Leitlinie implementiert?

Nach der Konsensuskonferenz wird die Leitlinie nochmals öffentlich zur Kommentierung ausgelegt. In dieser 6-8-wöchigen Phase kann jeder die Leitlinie einsehen und kommentieren. Die Kommentare werden gesammelt und alle bewertet. Es reicht von Hinweisen zu Tippfehlern bis hin zu substanziellen inhaltlichen Anmerkungen. Bei letzteren kann es der Fall sein, dass bei berechtigten Einwänden eine Empfehlung sogar nochmals komplett neu konsentiert werden muss. Nach dieser Kommentierungs- und Überprüfungsphase wird die Leitlinie durch die AWMF publiziert und ist somit gültig. Die Leitlinie ist unter anderem Grundlage für die zu erfüllenden Kriterien bei der Zertifizierung der Hautkrebszentren.

\section{Gibt es Instrumente zur Evaluation bzw. Umsetzung in der Praxis?}

Eine Form der Evaluation ist die Zertifizierung von mittlerweile über 40 Kliniken und Institute als Hautkrebszentren. Diese müssen Kriterien nachweislich erfüllen, die in der Leitlinie formuliert werden. Die Leitlinie, obwohl sie keine Richtlinie ist, wird auch bei Gerichtsverhandlungen immer als Referenz herangezogen.

\section{Dr. Eigentler, vielen Dank für das Interview!}

Kontaktadresse: PD Dr. Thomas Eigentler, Zentrum für Dermatologische Onkologie, Liebermeisterstraße 25, 72076 Tübingen, Deutschland, thomas.eigentler@uni-tuebingen.de. 


\section{Blickdiagnose}

\section{Welche Diagnose vermuten Sie?}

Ein 52-jähriger Mann stellte sich mit einer seit 20 Jahren langsam wachsenden, asymptomatischen Geschwulst unter der Nagelplatte des rechten Daumens vor. In der Untersuchung war die Geschwulst von weicher bis fester Konsistenz und entsprang aus der medialen Hälfte des Nagelbetts. Die darüberliegende Nagel-

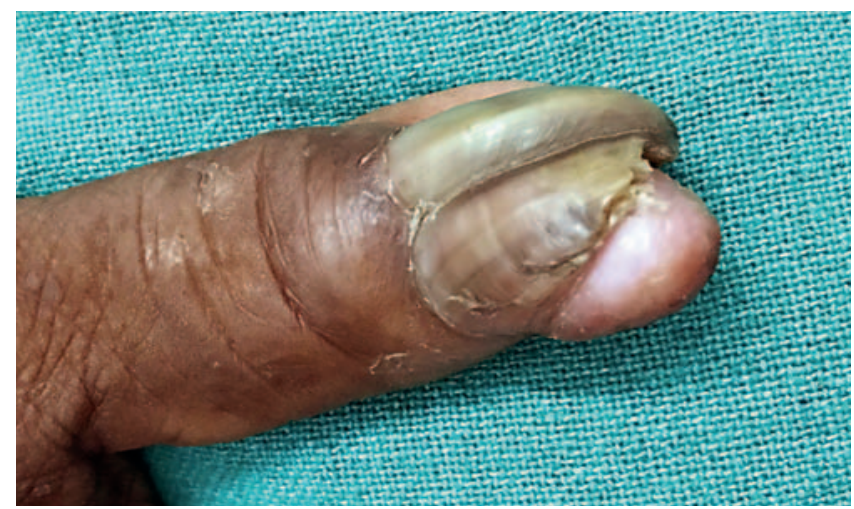

Abb. 1. Fleischfarbene weiche bis feste Geschwulst mit Ursprung in der medialen Hälfte des subungualen Bereichs des rechten Daumens, die zu Deformität mit gespaltenem Nagel führt. platte war onychogryphotisch und in der Mitte gespalten. Der mediale Anteil der Nagelplatte war vom darunterliegenden Nagelbett abgehoben (Abb. 1). Kein anderer Nagel war von einer ähnlichen oder einer sonstigen Erkrankung betroffen und die mukokutane Untersuchung lieferte keinen weiteren Aufschluss. In der bildgebenden Untersuchung zeigte sich ein Weichteiltumor ohne knöcherne Veränderungen (Übersetzung aus Kumar P, Tiwary AK, Barkat R, Alam G: Skin Appendage Disord 2018 (DOI: 10.1159/000489899)).

\section{Auflösung online unter: www.karger.com/blickdiagnose-kkd-4-2018}

\section{回微回}

Kontaktadresse: Dr. Anup Kumar Tiwary, Department of Dermatology and Venereology, House Number 12, Diamond Building, Government Medical College, Bareilly Road, Haldwani, Uttarakhand 263139, Indien, anup07tunnu07@gmail.com.

\section{Buchrezension \\ Willhellm Stolz et alı: Bildgebende Diagnostik in der Dermatologie}

Stuttgart, Thieme, 2018, 304 Seiten, 910 Abbildungen, 149,99 EUR

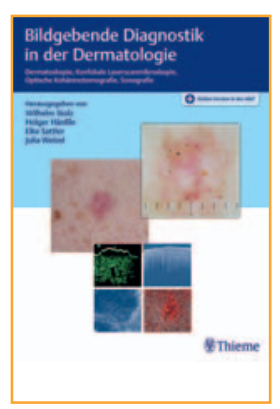

Der in diesem Jahr neu erschienene Atlas «Bildgebende Diagnostik in der Dermatologie», herausgegeben von Stolz, Hänßle, Sattler und Welzel, beleuchtet neben dem besonders bei Berufseinsteigern mit Hemmungen behafteten Thema Dermatoskopie auch weitere, teils neue Methoden der bildgebenden Diagnostik der Haut.

Der Hauptfokus liegt zunächst auf dem 180 Seiten umfassenden Kapitel «Dermatoskopie», welches auf dem Konzept des bisherigen Standardwerkes «Farbatlas der Dermatoskopie» aufbaut. Wie die Folgekapitel beginnt auch dieser Abschnitt mit einem Grundlagenteil, in dem zuerst Technik und Untersuchungsablauf erläutert werden. Danach werden dem Leser Schritt für Schritt die wichtigsten Diagnosekriterien der Dermatoskopie (Farbe, Begrenzung und Gefäßmuster etc.) anhand von eingängigen Schemata und klinischen Beispielbildern erklärt. Nach Erarbeitung der Grundlagen folgt eine systematische Abhandlung der unterschiedlichen melanozytären und nicht-melanozytären Hauttumoren, für deren Unterscheidung dem Leser ein Stufenalgorithmus an die Hand gegeben wird. Als eindeutige Stärke des Werkes sind hier die qualitativ hochwertigen Abbildungen hervorzuheben, in Folge derer der Atlas mit verhältnismäßig wenig Text auskommt. Didaktisch sinnvoll sind die makroskopischen, klinischen
Bilder den dermatoskopischen Bildern gegenübergestellt. Die weiteren, etwas kürzeren Kapitel sind den Themen «Sonografie», «Optische Kohärenztomografie (OCT)», «Konfokale Lasermikroskopie» und «weitere bildgebende Methoden» gewidmet. Im letzten Abschnitt «Methodenvergleich» werden die unterschiedlichen Verfahren in Hinblick auf ihre Vor- und Nachteile, ihre Einsatzmöglichkeiten und ihrem Potenzial, eine invasive Diagnostik in Einzelfällen zu ersetzten, gegenübergestellt. Besonders am Ende des Buches fällt die Beschriftung der Abbildungen jedoch recht spärlich aus, sodass es teilweise schwierig wird, Abbildung und Bildunterschrift in Einklang zu bringen. Für das Verständnis der Technik, der die unterschiedlichen bildgebenden Verfahren zugrunde liegen, sind ferner zumindest physikalische Grundkenntnisse Voraussetzung. Gut verständlich sind hingegen die anhand von über 850 Bildern dargestellten morphologischen Unterschiede der jeweiligen Hautveränderungen in den verschiedenen Untersuchungstechniken.

«Bildgebende Diagnostik in der Dermatologie» ist dank der hervorragenden Bilder und des strukturierten Aufbaus sowohl für Einsteiger als Lehrbuch, als auch für Fortgeschrittene als Nachschlagewerk im klinischen Alltag, sehr gut geeignet.

Clara Geppert, Mainz

Kontaktadresse: Dr. Clara Geppert, Hautklinik Universitätsmedizin Mainz, Geb. 401, 1. OG, Langenbeckstraße 1, 55131 Mainz, Deutschland, clara.geppert@unimedizin-mainz.de. 